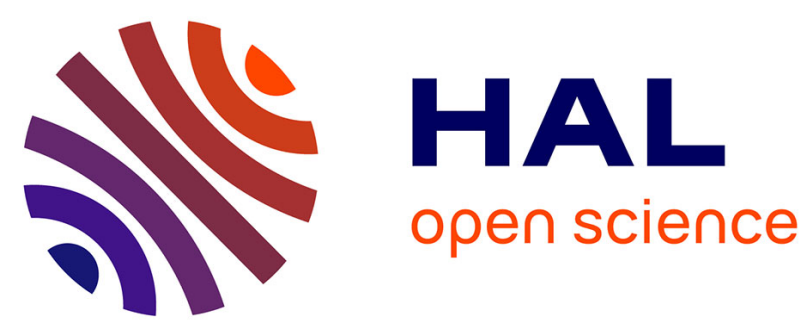

\title{
Towards "Avatar-Friendly" 3D Manipulation Techniques: Bridging the Gap Between Sense of Embodiment and Interaction in Virtual Reality
}

Diane Dewez, Ludovic Hoyet, Anatole Lécuyer, Ferran Argelaguet Sanz

\section{- To cite this version:}

Diane Dewez, Ludovic Hoyet, Anatole Lécuyer, Ferran Argelaguet Sanz. Towards "Avatar-Friendly" 3D Manipulation Techniques: Bridging the Gap Between Sense of Embodiment and Interaction in Virtual Reality. CHI 2021 - Conference on Human Factors in Computing Systems, May 2021, Yokohama, Japan. pp.1-14, 10.1145/3411764.3445379 . hal-03468547

\section{HAL Id: hal-03468547 \\ https://hal.inria.fr/hal-03468547}

Submitted on 7 Dec 2021

HAL is a multi-disciplinary open access archive for the deposit and dissemination of scientific research documents, whether they are published or not. The documents may come from teaching and research institutions in France or abroad, or from public or private research centers.
L'archive ouverte pluridisciplinaire HAL, est destinée au dépôt et à la diffusion de documents scientifiques de niveau recherche, publiés ou non, émanant des établissements d'enseignement et de recherche français ou étrangers, des laboratoires publics ou privés. 


\section{Towards “Avatar-Friendly” 3D Manipulation Techniques: Bridging the Gap Between Sense of Embodiment and Interaction in Virtual Reality}

\author{
Diane Dewez \\ Inria, Univ Rennes, CNRS, IRISA \\ Rennes, France \\ diane.dewez@inria.fr \\ Anatole Lécuyer \\ Inria, Univ Rennes, CNRS, IRISA \\ Rennes, France \\ anatole.lecuyer@inria.fr
}

\author{
Ludovic Hoyet \\ Inria, Univ Rennes, CNRS, IRISA \\ Rennes, France \\ ludovic.hoyet@inria.fr \\ Ferran Argelaguet \\ Inria, Univ Rennes, CNRS, IRISA \\ Rennes, France \\ ferran.argelaguet@inria.fr
}

\begin{abstract}
Avatars, the users' virtual representations, are becoming ubiquitous in virtual reality applications. In this context, the avatar becomes the medium which enables users to manipulate objects in the virtual environment. It also becomes the users' main spatial reference, which can not only alter their interaction with the virtual environment, but also the perception of themselves. In this paper, we review and analyse the current state-of-the-art for 3D object manipulation and the sense of embodiment. Our analysis is twofold. First, we discuss the impact that the avatar can have on object manipulation. Second, we discuss how the different components of a manipulation technique (i.e. input, control and feedback) can influence the user's sense of embodiment. Throughout the analysis, we crystallise our discussion with practical guidelines for VR application designers and we propose several research topics towards "avatar-friendly" manipulation techniques.
\end{abstract}

\section{CCS CONCEPTS}

- Human-centered computing $\rightarrow$ Interaction design theory, concepts and paradigms.

\section{KEYWORDS}

virtual reality, avatars, interaction, embodiment, guidelines

\section{ACM Reference Format:}

Diane Dewez, Ludovic Hoyet, Anatole Lécuyer, and Ferran Argelaguet. 2021. Towards "Avatar-Friendly" 3D Manipulation Techniques: Bridging the Gap Between Sense of Embodiment and Interaction in Virtual Reality. In CHI Conference on Human Factors in Computing Systems (CHI '21), May 8-13, 2021, Yokohama, Japan. ACM, New York, NY, USA, 14 pages. https: //doi.org/10.1145/3411764.3445379

Permission to make digital or hard copies of all or part of this work for personal or classroom use is granted without fee provided that copies are not made or distributed for profit or commercial advantage and that copies bear this notice and the full citation on the first page. Copyrights for components of this work owned by others than ACM must be honored. Abstracting with credit is permitted. To copy otherwise, or republish, to post on servers or to redistribute to lists, requires prior specific permission and/or a fee. Request permissions from permissions@acm.org.

CHI '21, May 8-13, 2021, Yokohama, Japan

(C) 2021 Association for Computing Machinery.

ACM ISBN 978-1-4503-8096-6/21/05 . .\$15.00

https://doi.org/10.1145/3411764.3445379

\section{INTRODUCTION}

$3 \mathrm{D}$ object manipulation is an essential interaction task in virtual reality (VR) [14], from industrial/medical training to artistic/modelling applications. It encompasses multiple subtasks, from object selection, translation, rotation to scaling [15]. Numerous manipulation techniques have been proposed in order to cope with the limitations of VR systems and the complexity of 3D manipulation, such as virtual hand techniques enabling direct manipulation, or raycasting techniques allowing out-of-reach manipulation [15, 69]. One important component of such techniques is the feedback provided to users, which provides information on how their actions influence the manipulation task. However, what happens when the user is embodied in a virtual body (i.e. an avatar) while performing the manipulation task? Avatars are a subset of user representations that may be anything from a single body part (e.g. hands) to a full body. Full-body avatars are becoming ubiquitous in VR applications, especially for social VR. This has stimulated research on how users can embody such avatars, i.e. feel like avatars are their real bodies. This "sense of embodiment" [50] can improve user experience, it can change cognitive performances [7] or the way users interact [49]. Thus, the following questions arise: can the user representation influence the manipulation process? Can manipulation techniques influence the perception of the user's representation?

When manipulating objects, the avatar provides feedback regarding the users' motions which can influence perception of their motions [75] and even the environment [47]. This could disrupt the manipulation task and impact performance criteria such as speed or accuracy. For example, a 3D hand model outperforms a whole arm in pointing tasks [111], thus a more complex user representation might be more disturbing than helpful. Reciprocally, the design choices regarding control and visual feedback of the manipulation technique can modify users' perception of their avatar. For instance, the Go-Go technique enabling out-of-reach manipulation gives the illusion of a very long arm, which can affect embodiment [25]. If we want to make choices that ensure embodiment, these may contradict choices made to increase performance. Depending on the use-case, it may be preferable to maximize embodiment or performance, or ensure an adequate level of both. Thus, there is a need for investigating how to both maintain efficiency of a manipulation 
technique when an avatar is used, and how design choices impact the sense of embodiment.

This paper unites the knowledge on manipulation and avatar embodiment. It extends the review by Seinfeld et al. [95] on the cognitive concepts behind the use of avatars in Human-Computer Interaction (HCI) through a focus on manipulation tasks and provision of concrete guidelines. It also sheds new light on previous works on manipulation techniques such as the survey by Medeiros et al.[68] and aims to help improve current techniques and develop new ones. First, we identify several design issues raised by the use of avatars during manipulation, from the dual perspectives of how avatars impact common interaction criteria, and how design choices impact embodiment. This analysis leads to several practical guidelines for designing avatar-friendly manipulation techniques, i.e. techniques which take the user's avatar into account in the design process to preserve both user performance and sense of embodiment. This paper ends on a discussion and several leads for future research. The main contributions of this paper are:

- an analysis of how avatars impact manipulation tasks

- an analysis of how the manipulation design choices impact the sense of embodiment

- a comprehensive set of guidelines based on our analysis to design avatar-friendly manipulation techniques

- several research topics for remaining open questions

The remainder of the paper is structured as follows. Section 2 overviews the global structure of our meta-analysis. Section 3 explains some advantages and drawbacks of using avatars in VR applications, which explain most current design choices. In Section 4, we take the opposing point of view by reviewing the potential impact of design choices on embodiment. We then propose practical guidelines in Section 5, classified into three categories: input devices, control and feedback. Finally, Sections 6 and 8 provide discussion about the need of avatars for manipulation, as well as future research topics (RT).

\section{TOWARDS “AVATAR-FRIENDLY” 3D MANIPULATION TECHNIQUES}

3D manipulation tasks encompass all the modifications that can be done on a virtual object, often summed up to four canonical tasks: selection, positioning, rotation and scaling $[15,82]$. If we model the manipulation task using an action-perception loop (see Figure 1), users' actions are captured by the input devices, those actions are processed by the manipulation technique which controls the state of the interaction, then feedback is generated and perceived by the user which enables the user to decide the follow-up actions. If we take the example of the virtual hand metaphor [71, 84], users can grab a virtual object by performing a reaching motion and then pressing a button on a controller. These actions would be mapped to a virtual hand which will grasp the virtual object and provide visual feedback to the user. The manipulation technique might also include additional feedback, e.g. highlighting the object to show the outcome of the action (the object has been selected) [3].

These different steps can be visualised in an action-perception loop summarized in Figure 1. In this figure, we can notice the importance of the avatar, i.e. the user's virtual representation driven by his/her movements. It acts as the user's proxy in the virtual environment (VE, i.e. the 3D space in which the user is immersed) and provide both control and feedback. The avatar is therefore not just a 3D model in the VE, but an entity through which the user can interact with the VE. However, the avatar does not only enable interaction with the VE, but becomes the user's virtual representation in the VE, influencing how the VE is perceived and even generating the illusion that it is the user's real body. This phenomenon, called the sense of embodiment, can be defined as "the sense that emerges when a body's properties are processed as if they were the properties of one's own biological body" [50]. It can be split into three subcomponents: the feeling of being at the same location as the body (sense of self-location), the feeling of being in control of the body (sense of agency) and the feeling of owning the body, that it is a part of one's own real body (sense of ownership) [50]. Different factors can influence these subcomponents. For instance, the perspective from which users see their avatar (first or thirdperson perspective) can influence the sense of self-location [33, 37]. Multisensory congruence can influence all the subcomponents: the sense of agency [33], the sense of self-location [57] and the sense of ownership [54]. Some of these factors influence more than others the global sense of embodiment [28]. This virtual embodiment is similar to effects observed in real-world experiments like the rubber-hand illusion $[12,112]$, in which people can appropriate a rubber hand thanks to synchronous visuotactile feedback. Similarly in VR, multisensory stimulation is an effective way to elicit embodiment [99]. Synchronous visuotactile or visuomotor stimulation have been shown to create a sense of embodiment [54, 98], with visuomotor feedback most contributing to this effect. However, when the coherency between users' actions and perception is broken (e.g. asynchronous visuomotor or visuotactile stimulation) the illusion can be broken [54]. The main objective of this paper is to integrate the notion of avatar in the design process of 3D object manipulation, focusing not only on usability and performance but also on users' perception of their avatar (i.e. the sense of embodiment). The following sections discuss the considerations for designing such "avatar-friendly" techniques. First, Section 3 presents the current state of manipulation tasks using avatars. It mainly focuses on performance and does not take embodiment into account, as it is not currently sufficiently considered in interaction design. For this analysis, we reviewed papers using performance or usalibity criteria that included avatars in their protocol. On the other hand, Section 4 deals with the cognitive aspects and the perception of the avatar depending on the techniques design choices. For this part, we reviewed mostly papers on the sense of embodiment, in which manipulation tasks were performed or specific design choices were made (for example, adding haptic feedback). In both sections, links are made to the guidelines based on the literature (detailed in Section 5) or to the research topics (presented in Section 6) when there is not enough literature to justify a guideline. The guidelines are classified into the three main categories of design choices: input devices (ID), control and mapping (CM) and feedback (FB).

\section{THE AVATAR AND ITS IMPACT ON MANIPULATION}

The avatar can be seen as a tool that informs users of their position in the VE and enables them to move and interact with this environment [95]. The avatar therefore helps users during manipulation 


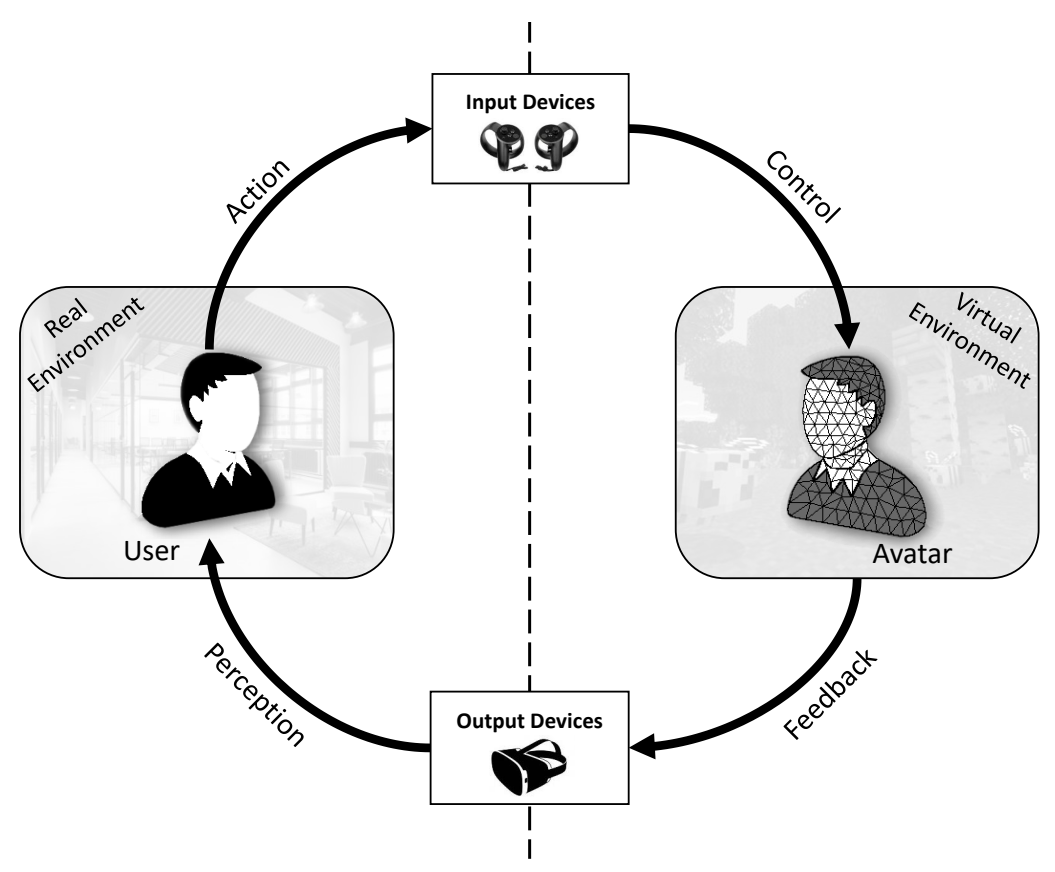

Figure 1: Action-perception loop: the user can control the avatar thanks to the input devices, so as to interact with the VE. In response to the user's actions, the VE provides feedback perceived by the user thanks to the output devices.

by providing spatial awareness [23] and can therefore increase performance [66]. Avatar appearance can vary in terms of shape, size or texture. The more complex the representation, the more information users can obtain to potentially improve their performance. For instance, people perform better in a bow-shooting task with a full-body, and tend to prefer this representation over other simpler representations (only controllers or floating hands) [34]. Another study found a user preference for performing a tool-based pick and place task with a visual hand representation, albeit performance was similar to the condition of performing the same task without a visual hand representation [90]. In terms of realism, a visually faithful avatar does not outperform a generic avatar in a cognitive manipulation task [60] or a robot avatar in a pointing task [94], showing a potential low impact of human-likeness on performance. Avatars can also enable certain illusions to facilitate interaction, e.g. elicit weight sensations [22, 45], when the Control-Display (CD) gain is modified. But avatars can also have drawbacks and negatively impact the manipulation. For example, they can generate occlusions if they are too large and opaque, or seen from a thirdperson perspective (i.e. the viewpoint is not co-located with that of the avatar). In this section, we will mostly take the designers' current point of view by studying how the avatar can impact manipulation. This impact can motivate, or on the contrary, discourage the use of avatars.

\subsection{Avatars Can Alter Distance and Object Size Perception}

In real life, the body serves as a reference frame to interact with our surroundings [39]. It is even more essential in VR, as users tend to show a size and distance perception bias, usually underestimating distances and object size [80]. However, several authors have demonstrated that displaying an avatar can improve these perceptions [73]. Similarly, it was also found that hands with a personalised size increase the correct estimation of object size [47], and that this effect can also depend on the realism of the avatar's appearance [74]. This is coherent with early results showing individual differences during manipulation of virtual objects and the importance of calibrating the experience for each individual [114] (see guideline FB1).

\subsection{Avatars Can Be a Source of Visual Occlusions}

Occlusions often serve as a depth cue, helping us compare depth between objects [77]. However, the avatar can occlude virtual objects and therefore hinder their manipulation. The more body parts are represented, the higher the risk of this occurring. This can prove critical in applications providing only visual feedback contrary to real life, where other types of feedback (haptic, auditory) can prevent us from being bothered by occlusions. For example, a study has found that users perform better with a virtual hand than with a virtual whole arm [111]. The authors hypothesise that this is due to the whole arm hiding the scene more and creating occlusions. This is also consistent with the study by Argelaguet et al. [4], where users performed better with unrealistic hands like skeleton hands than with realistic hands which generate more occlusions (see Figure 2).

In order to minimize occlusions, some potential solutions can be considered. For example, the avatar's hands can become transparent $[16,119]$ when users approach a virtual object. This maintains an anthropomorphicrepresentation of the user while keeping the 


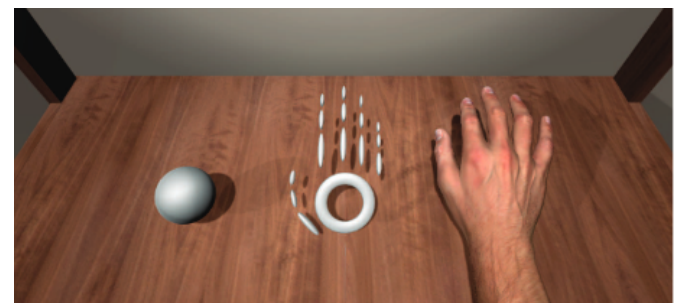

Figure 2: Examples of different possible hand appearances for a manipulation task. Image from [4]

virtual object visible through this representation (see guideline FB3). Providing additional feedback of the object hidden by the avatar (e.g. with a supplementary view from another perspective) [10] is also an alternative. The latter has the advantage of not changing the appearance of the avatar at the exchange of higher task complexity.

\subsection{Avatars Can Increase the Tolerance to Anisomorphic Manipulation}

Manipulation can be isomorphic, in which case the translations and rotations applied to manipulated objects in the VE strictly correspond to the ones applied to the input device. But, it is also possible to apply a gain to users' input. This gain is called the control-display gain, i.e. the gain applied to the input to compute the position of the displayed representation, and has been studied in the 2D [21] and 3D contexts [3]. This gain can be constant or adaptive (it varies depending on some conditions). A CD gain lower than 1 means the displayed motion is smaller than the real motion. It can be used to provide a higher control for precise positioning [27]. A CD gain greater than 1 means that users' motion is amplified, which can be helpful to provide higher speed of manipulation when precision is not crucial, or to reach remote objects [83]. Creating distorted motion by modifying the $\mathrm{CD}$ gain can also be used to provide haptic feedback, to lead the user to a physical prop $[6,53]$. This technique applies an offset to the virtual hand so the user will reach the real tangible object.

Thanks to the avatar, it is possible that users do not notice the motion distortion. Users' visual perception dominates proprioception [19], making this illusion possible. For example, even if users perform a small hand movement in real life, if their avatar moves its hand a lot, they will trust the visual feedback more and think that they performed a larger movement. Several studies investigated the thresholds beyond which users notice this modification of their movements. It was found that displaying a realistic hand instead of a spherical cursor increases the detection thresholds during remapped movements by more than 30\% [75] (see guideline FB7). When reaching for an object, participants did not detect the horizontal shift applied to their movement as much in the realistic hand condition as in the spherical cursor condition. Another study found that the subjects who feel a sense of ownership towards their avatar tend to detect distorted motion less [18]. The influence of avatars with different levels of realism and visible body parts on the detection of remapped motion should be further investigated (see research topic RT1).

\subsection{Coherency between Avatars and Input Devices Can Influence Manipulation}

There is a strong relationship between the chosen user representation and the input device used [95], e.g. it is rare to use hand tracking to control a simple sphere cursor. The user representation can also impact the way users hold the input device, how they manipulate objects [48], as well as their preferred input device. For instance, previous work demonstrated that users prefer hand tracking to controllers when represented by realistic virtual hands because it is more realistic and fun [59, 72]. However, designers usually choose controllers in most applications, probably because they are more reliable, accurate [72] and commonplace. Various types of controllers exist, differing in terms of shape, buttons and other inputs available, weight or haptic feedback provided. It is questionable whether the way they are displayed in the VE should be generic or not. Using controllers therefore raises several questions: When using such controllers for manipulation, what user representation should be chosen? But also, how does this choice impact the interaction performance? With most controllers, a virtual hand can be used as a generic representation that would directly manipulate virtual objects. In 1999, Bowman [14] recommended the use of virtual hands rather than virtual tools like raycasting to ensure efficient positioning and rotation of virtual objects. In this case, the different discrete inputs (buttons on the controllers) can launch actions such as grabbing of virtual objects, a solution often used in current VR applications. But sometimes, the controller used is inspired by a real tool and specific to an application, especially in training applications. In this case, it might be preferred to display the 3D model of this tool in the avatar's hand, to provide realism and coherency with the haptic feedback provided by the input device [44] (see guideline FB5). Using virtual tools in the same manner that we use real tools has the advantage that it is straightforward. Moreover, using virtual tools was found to activate the same regions in the brain as real tools [88], which highlights that virtual tools are treated behaviorally by the brain in a similar manner to physical tools. Displaying virtual tools instead of hands can also be an explicit cue for possible actions in the VE [95]. Choosing a virtual hand or a virtual tool in the avatar's hand as the representation of an input device can therefore change the way the user's interact. This impact of the input device display in the VE, in relation to the avatar, should be further explored (see research topic RT2).

\section{INTERACTION DESIGN CHOICES AND THEIR IMPACT ON AVATAR EMBODIMENT}

In the previous section we studied how avatars can influence manipulation. However, this design problem can also be studied the other way around, by focusing on how design choices of the manipulation technique impact users' perception of their avatar, more specifically their sense of embodiment. Although different implementations can be proposed for each manipulation paradigm, some variations of a chosen technique can be more adapted than others when a virtual body is introduced in the VE. In this section, we review the current work on embodiment and interaction that could justify design choices for avatar-friendly manipulation techniques. The analysis is decoupled considering the three main design choices for 
a manipulation technique: the input device, the control mechanism and the feedback.

\subsection{Input Devices}

Numerous input devices exist, varying for instance in shape, Degrees of Freedom (DoF), input modality and input interfaces (e.g. buttons, joysticks). If the user has a virtual representation, it means the input device must ensure both the manipulation but also the control of his/her virtual representation. While the input device is usually the same for both avatar control and manipulation such as the case of the virtual hand metaphor, hybrid methods are beginning to emerge using different input devices for avatar control and object manipulation (e.g. hand tracking for hands control and a tablet for manipulation [102]).

4.1.1 Shape. Controllers have various shapes, but most of them are held in a power grip. When the shape resembles a real tool, this provides high interaction fidelity. Input devices adapted to the task can give better performances $[9,79]$ as the way the user hold $s$ them is more coherent with the task to perform [8] (see guideline ID1). Little work exists regarding the influence of device shape on embodiment, probably due to the various types of controllers and its potential low impact. However, there could be an impact of the shape on embodiment, especially depending on the associated virtual model. Depending on the way users hold a device, it can change the way they control their representation and manipulate objects, which can make them feel more or less in control. In particular, this may impact their perception of their avatar if the manipulation is not intuitive. More importantly, depending on the shape, it is crucial to choose an adequate mapping between the controller and the actions performed in the VE (see research topic RT2).

4.1.2 DoF. For most manipulation techniques, the virtual hands are the tool to interact with the virtual objects, except when there is a use of an intermediary element like the ray in raycasting techniques. When using a virtual hand, the DoF provided by the input device are used mostly to control this hand. Intuitively, we can think that the more DoF provided for hand control, the higher the level of control and the sense of embodiment. Supporting this theory, hand tracking was found to elicit a higher sense of ownership than controllers, probably because it provides a finer control of the users' virtual hand [59].

However, this effect can also depend on the DoF provided by the user representation compared to the DoF provided by the input device. For example, while Argelaguet et al. [4] found that agency was better with unrealistic hands (sphere and skeleton hand, so with limited DoF) using Leap Motion, Lougiakis et al. [61] found no difference between different hand appearances with a similar protocol using controllers. A potential explanation for this could be that controllers offer less DoF and thus reduce expectations in terms of hand control. The more DoF are provided by the input device, the more control users want during manipulation, especially when realistic representations are used [4]. These results suggest that more research should be done on combinations of different input devices and avatars during 3D manipulation (see research topics RT2 and RT3).
4.1.3 Input Modality. In order to track the users' hands, two main input modalities could be considered: full-hand tracking and controllers. While controllers are efficient and commonly used, manipulating objects directly with the hands remains the most realistic way both of interacting and of controlling the avatar. It allows fine manipulation as well as direct control of the virtual hand. This is possible thanks to optical devices (Leap Motion, Kinect) or wearables (mostly gloves). However, it implies having precise and robust finger tracking. As previously discussed, in theory hand tracking offers more DoF and therefore better control and probably a higher embodiment [59] (see guideline ID2).

However, imperfect tracking can cause mismatches between the visual display and the users' real actions. When the hand movements are constrained by the controllers, there are fewer chances for users to notice mismatches with their real hands. Experiments in which finger tracking provides a good sense of embodiment are usually constrained experiments, for example requiring participants to keep their palm facing down [42]. Therefore, constraining movements could be one possible solution. Recent input devices (e.g. Oculus Touch, Valve Index) use capacitive sensors for the fingers, providing a good trade-off between offering the efficient tracking of a controller and more precise finger movements for manipulating objects. It would also be interesting to study the impact of hybrid input [43] (using both a controller and hand tracking) on the sense of embodiment (see research topic RT3).

In addition to reliability, controllers also often offer buttons as input, which can be useful to trigger actions in the environment and feel in control, instead of for example launching an automatic animation of grabbing an object when the hand is near it. Using buttons offer low-fidelity interaction, but this can be better in terms of performance and acceptance than moderate-fidelity solutions [65]. The best scenario still remains the high-fidelity case, where the user performs the entire action like in the real world, as a complete control of the avatar elicits a higher sense of embodiment than just triggering actions [28]. More important than the input modality itself, what matters is the mapping applied to this input (see research topic RT2).

\subsection{Control}

A great part of manipulation techniques do not consider a 1:1 mapping between input motion and the resulting manipulations. For example, modifications of the $\mathrm{CD}$ gain or constrained motions are commonplace in order to increase the precision of free-hand manipulation. However, such control mechanisms introduce mismatches between the actions performed by the user and the motions of their avatar. As visuomotor coherency is one of the major inducers of the sense of embodiment [54], such changes in control could have a negative impact in the overall sense of embodiment.

4.2.1 Motion and Manipulation Constraints. Constraints can be used on manipulation to interact more quickly or respect real world physics. Most constraints applied during manipulation are meant to avoid collisions, or to reduce DoF (limit the manipulation to a particular surface or an axis for example). When constraining the manipulation, it means that the avatar's motion is also constrained, which can create a visual-proprioceptive mismatch and disturb the senses of agency and self-location [86]. First, handling collisions 
might seem a good idea to create a realistic environment that respect the laws of physics. For instance, when moving an object on a table, it is possible to apply semantic constraints based on real-life physics, as long as the motion of the user is coherent with this constraint (e.g. a horizontal motion on the surface of a table). However, especially for wide and fast motion, blocking the avatar outside objects can create huge offsets between the real hand and its virtual counterpart This will probably cause a break in the sense of self-location but also ownership, which is sensitive to spatial location [89]. Therefore it is better to do not handle collisions for this type of movements (see guideline CM1). If users experience a high sense of presence (i.e. the sense of being in the VE), they should intuitively avoid collisions, especially when using a realistic avatar [76]. For finer motion, handling collisions is recommended, as it is preferred by the users (see in Section 4.2.4).

Second, constraints separating DoF (for example to move precisely an object on one direction) can also create big mismatches between the user's position and the avatar's one. In such a case, it is preferable to use explicit constraints. They can be explicitly defined by the users so they know what motion they can perform or not [40]. It is also possible to use gestures to determine customised constraints [35], which has the advantage of making users move their avatar and potentially appropriate it. To reduce DoF, the classical use of widgets (see an example on Figure 3) explicitly constrain the manipulation, which can be more predictable and impact less the sense of agency. Widgets can be seen as virtual objects that the user can use to indirectly manipulate objects. Common constraints on the manipulation still apply to the widgets though, for example the grabbing of the widget tool must seem realistic (see Section 4.2.4). While widgets are intuitive, they do not provide high-fidelity interaction using directly the avatar. New techniques of DoF separation adapted to the avatar could be invented (see research topic RT4).

Another solution to handle constraints could be to dissociate the avatar, that provides a realistic representation of the user, from a second form of representation that is not affected by the constraints. It can be the tool used, or a ghost [116] or skeleton hand [4].

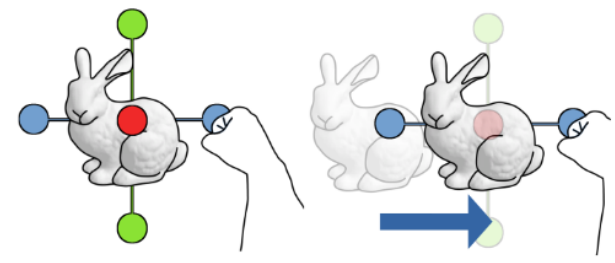

Figure 3: Example of a widget to constrain manipulation. Image from [70]

4.2.2 Remapped Motion: Modification of CD gain. Avatars provide a direct visual feedback of the user's position and motion in the environment. If the $\mathrm{CD}$ gain differs from 1, this relation between the user's motion and the avatar's one is altered and can impact the sense of embodiment. Typically, manipulation methods employ either constant of adaptive gains.
Constant gains. The higher or lower is the CD gain, the higher will be the mismatch between the avatar's hand and the user's real hand. Although visual feedback dominates proprioception [19], and people do not notice easily motion distortion (i.e. when their motion is amplified or decreased when achieving a task) [32] and can even adapt their motion to this distortion [13], if the mismatch is too important it can frustrate the user and break the sense of embodiment. A study found that while the senses of agency and ownership are quite resistant to visual-proprioceptive mismatches (induced by a constant offset between the real and virtual hand), the sense of self-location is very sensible to them [86]. Moreover, Kokkinara et al. [55] found that when users' movements are amplified, this affects their perception of the VE and agency (contrary to the study by Pritchard et al. [86]), but not ownership. When the user's motion is distorted and it creates a mismatch that is noticeable, the user can therefore feel out of control and the sense of agency can be affected. To avoid this, it is better to do not apply gain, especially on fast and wide movements because it would quickly create a large mismatch between real and virtual hand (see guideline CM2). As discussed in Section 3.3, realistic hands make remapped motion less noticeable [75] (see guideline FB7). Furthermore, instead of modifying the virtual body's motion, the distortion can also be applied on the virtual world [6]. This is the same principle as redirected walking, the world is translated when users move their head. This is only adapted to manipulation tasks necessitating head motions. A hybrid solution mixing world and body movements distortion could be a good solution to avoid the drawbacks of both techniques.

Adaptive gains. The gain applied could also be adaptive, i.e. its value can vary depending on the conditions. For example, the gain can depend on the object distance to the user, closer manipulation necessitating a lower gain to be precise, and further manipulation a higher gain [78]. It was found that the acceptance of distorted motion is increased when the distortion is gradually introduced [81]. Altering the motion gain slowly in the context of physically tiring tasks still elicits a sense of ownership [26]. When distortion must be used in an application, it is better to adapt it depending on the situation (see guideline CM5). Different gains could also be applied depending on the direction of the movement, as users' notice more easily offsets on the horizontal axis than in other dimensions [24].

A preferable solution to avoid the impact of distorted motion on embodiment could be to decouple the tool from the body. An isomorphic control would be kept for the avatar, while the manipulation would be anisomorphic [35]. The gains would only be applied on the tool, thus not affecting the avatar itself, for example in out-of-reach interaction (see research topic RT5).

4.2.3 Out-of-reach Manipulation. A special type of manipulation that can create a strong mismatch and potentially impact embodiment is out-of-reach manipulation. In the space in which we can reach objects, i.e. our peripersonal space, the challenge is mostly focused on the range of motion, the hand poses, the feedback of actions, or the display of input device. However, outside the peripersonal space, it is necessary to choose how to represent the action, and how it impacts the avatar. Common out-of-reach manipulation techniques are the Go-Go [83] and the raycasting techniques [11]. 
Go-Go technique. The Go-Go technique applies a non-linear CD gain to the user's input. The more users extend their arm, the further the virtual hand goes. If the limit of CD gain is too high, the mismatch can be important between the user's real hand position and its virtual counterpart. This could potentially impact all the sense of embodiment components. Several choices of feedback can be considered in this problem [25], but all of them can highly impact the sense of embodiment. In the case of full-body avatars, the hand could be detached from the virtual body. However, it has been found that body discontinuity reduce body ownership but does not necessarily impact motor performance [96]. A hand attached to the arm by a rigid wire disturbs embodiment but not skin conductance in reaction to a threat [110]. A potential solution could be to have an extensible arm, which can maintain a correct sense of embodiment, until reaching a certain length [51] (see guideline FB4). Other feedback can be imagined, like a robotic extensible arm, a ghost clone of the hand going towards the object (as already used in training [116]), or an outside assistance like a drone going to the object instead of the user's body. It depends on the context of the application, if the realism must be maintained or not.

Raycasting technique. The raycasting technique originally comes from the pointing technique [11] with an additional ray to provide visual feedback of selected objects [71]. It has the advantage of being body-referenced but not part of the avatar. However, depending on the way it is represented, it may impact ownership by providing an unrealistic feedback. If we consider the basic raycasting in which the ray is linked to the hand, we can display the ray coming from a finger, probably the index because it is the finger associated to pointing. We could also display a laser pointer in the avatar's hand instead, to maintain realism. The choice can also be impacted by the device input, if we use hand tracking we might prefer the first option, while the second could be better when a controller is used, or even a tracked stylus [108]. Other raycasting techniques exist, referenced to other parts of the body, for example to the head. In this case, a virtual headlamp could be used as a more coherent feedback.

The two techniques both have their pros and cons. The Go-Go technique uses direct manipulation, which is more intuitive and uses the avatar to appropriate it. The raycasting technique might be safer for self-location and agency, as it does not modify the CD gain and the virtual body does not have to be modified or reshaped to use this technique. More variants of feedback for out-of-reach techniques and their impact on embodiment should be investigated (see research topic RT5). The ideal would be to invent a technique that is as intuitive as the Go-Go technique but does not have to modify the avatar.

4.2.4 Control of Dexterous Hand Interactions. Grabbing virtual objects is probably one of the hardest challenges in manipulation. Because of the lack of haptic feedback in VE, if a 1:1 mapping is applied to hand movements, visual artifacts can appear: unrealistic hand poses or fingers passing through objects. This can affect embodiment. However, it is known that humans are more sensible to visual interpenetration than proprioceptive offsets [19]. They tend to believe more what they see than what their proprioception tells them. Designers therefore often choose to handle collisions and to define hand poses on the virtual objects, so the user is not disturbed by interpenetrations. This solution is usually preferred by the users, even though it can decrease performance [85]. But, if users feel too constrained by the collisions, this may impact the sense of agency and self-location. This also applies for defined hand poses: if they are too different from users' real poses, this could affect embodiment. People usually tend to adapt to the visual feedback provided, and can be disturbed for example when the input action required is different from the feedback. This was noticed for example in the experiment by Lok et al. [60] in which participants had to do a pinch movement but observed a grasping animation. Several participants started performing a grasp movement. Such adaptation needed might potentially influence the sense of embodiment.

The preferred feedback during the grasping of a ball has been studied, which confirms that handling collisions for fine manipulation is preferred [20] (see guideline CM4). Participants preferred visualising their hand outside the object, even though their real fingers were actually entering the sphere. This condition preserved the sense of ownership. Providing complete control of the virtual hand does not always seem to be the best choice.

Assistance in taking virtual objects is often used to make the manipulation more intuitive and realistic. This can be used without affecting agency and ownership [81] (see guideline CM3). However, if too few hand poses are provided, users may feel limited in their actions, which could impact the sense of agency. If users can grab an object even with unrealistic hand poses, the application will lack realism. Since defining many hand poses in a VR application can be time-consuming, it would be interesting to study their influence on the sense of embodiment, especially agency (see research topic RT6).

\subsection{Feedback}

The feedback provided to the user while performing an action can also impact embodiment. First the avatar itself is part of the feedback and can impact embodiment; for example if it is modified during manipulation. However, the type and characteristics of the feedback provided during an action can also affect embodiment.

4.3.1 Avatar Appearance During Manipulation. The avatar is the main source of feedback during motion in the VE. To increase usability or performance, its appearance can be modified during manipulation (see section 3.2). However, these changes must be done carefully. Even though avatar appearance is not the most important factor in the sense of embodiment [28], several studies showed its importance in inducing a good sense of ownership [113]. Designers must be aware that modifying it can impact how much users feel like the avatar is part of their real body. For example, the avatar's hands can be made transparent to avoid occlusions. But, if the hands are too transparent, this might impact the sense of ownership [64] and agency [16]. It is also possible to use skeleton hands [4], which provide a good sense of agency but a lower sense of ownership than realistic hands. To ensure a good sense of ownership, it seems important to keep a realistic avatar. Transparent hands seem to offer a good trade-off, as long as the hands are not too transparent (see guideline FB3).

When a tool is necessary for manipulation, or when a controller is used as input device, the feedback at hands level can be either 
the hand (controlled by the input device, for example by mapping buttons to the movements of the hand) or the tool held in the hand. The feedback chosen and its coherency with the input device used might impact embodiment. While some studies start investigating this question [2], this topic needs further research (see research topic RT2).

4.3.2 Perspective. Perspective is the user's point of view on the VE. Perspective is important to perform an action precisely, especially when the user can only rely on visual feedback. In applications using an avatar, various camera positions can be used, each of them providing a different amount of visual information. Perspective is generally described as a third-person perspective (the viewpoint is outside the user representation) or a first-person perspective (the user representation is co-located with the real body). A thirdperson perspective (3PP) is usually advised for navigation because it provides a better spatial awareness, but some recent work found that a first-person perspective is actually preferred [67]. For manipulation, a first-person perspective (1PP) is usually preferred and more precised $[37,92]$ as the user can directly manipulate objects like they would in real life. Moreover, 3PP requires an additional cognitive load for considering both the transformation users want to apply to a virtual object and the offset between their vision and their avatar.

In the literature on the role of perspective in the sense of embodiment, it was found that a $1 \mathrm{PP}$ is preferred to elicit embodiment. It has been found that subjects experience a higher sense of ownership from a 1PP, even though it can be experienced from a 3PP to some extent with a synchronous motor feedback [33, 37]. The sense of agency seems less impacted by the perspective, and can be felt either from 1PP or 3PP as long as the movements are synchronised. A potential solution, more complicated to implement, would be to have both perspectives [93].

In conclusion, it is better to have a $1 \mathrm{PP}$ to have both an efficient manipulation and a high level of embodiment (see guideline FB2).

4.3.3 Haptic Feedback. Visual feedback is the main feedback provided in VR. However, in real life, people receive other types of feedback that help during manipulation. Additional feedback like haptic feedback [41] can also be provided in VR and can impact manipulation performance [104]. Haptic feedback can be provided through tactile feedback (touch, to feel the object texture for example) or force feedback (e.g. users feel a resistance when they collide with an object) [17]. There are nowadays output devices that are put directly on the body [100] that provide vibrotactile feedback and can make the user more aware of their virtual body. Synchronous visuotactile feedback provided by an experimenter was one of the first methods used to elicit embodiment towards a virtual body [98]. If users feel a touch and observe at the same time a stimulation on the virtual body that can cause this touch, the brain interprets that the virtual body must be their body. A study found that haptic feedback provided by wearable devices can increase the sense of embodiment [30], especially force feedback. In addition of the information it provides during manipulation, haptic feedback is therefore also a way of increasing the sense of embodiment and should be used when possible (see guideline FB6).

When it is not possible to provide haptic feedback, pseudo-haptic feedback can be used. This technique can create an illusion of weight by modulating the gain applied to users' motion [45]. However, this should be used with precaution when using avatars as it can create a mismatch between users and their avatar (see Section 4.2.2).

4.3.4 Auditory Feedback. Another type of feedback that can be provided to users is auditory feedback. Auditory feedback provides important information when manipulating objects in real life. Like haptic feedback, it can inform us of collisions, it can also help in perceiving textures [56]. However, its relation with the sense of embodiment has been less studied than other types of feedback. The major study on auditory feedback and virtual embodiment is from Tajadura et al. [105] who found that we can enhance embodiment of a child's virtual body when the avatar's voice and appearance are coherent. One study investigated the role of auditory cues in the rubber-hand illusion. Synchronous auditory feedback seems to strengthen the illusion [87]. With a task of clapping hands in a non-immersive VE, no significant effect of the auditory feedback on virtual body ownership was found, showing a potential low impact of this type of feedback in the ownership illusion [62]. Several studies exist on the link between body perception and sound [5]. For example, when the sound heard when a small hammer hits a person's hand is the sound of a hammer hitting a piece of marble, it can give the person the illusion that the hand is made of marble [97]. Because the sound informs the user of a contact with an object, it can also be used to create a long arm illusion [106]. However, to our knowledge, no study has been done on the impact of auditory feedback during manipulation on the sense of embodiment. The impact of different types of feedback during object manipulation is still an on-going domain of research (see research topic RT7).

\section{GUIDELINES}

VR stakeholders usually design applications based on their instinct, or empirical experience, because of a lack of simple design advice, in the form of guidelines or claims for example. However, proposing and validating design guidelines for VR is already a challenge in itself [115]. There are too many factors impacting interaction, and too many individual differences, that make generalization from experiments very challenging. A set of guidelines for designing VR applications has been proposed by Gabbard [31], already talking about user representation. For instance, these guidelines recommend to use an efficient embodiment (provide enough sensory information) and let users control their appearance in the VE. But, these guidelines are general and not precise enough to design manipulation techniques. Guidelines for designing 3D manipulation techniques have also been proposed by Bowman et al. [15], however, they do not consider user representation. Other guidelines, heuristics and claims [103, 107], more or less general, have been proposed since, but the avatar is usually forgotten. Guidelines specific to manipulation, considering the avatar, would help VR developers and encourage the use of avatars. We propose in this section design guidelines, justified by existing literature, that can be used in early stages of a design pipeline. These guidelines are focused on design choices for manipulation techniques involving avatars, therefore we refer the reader to the guidelines of Gabbard [31] concerning the remaining of the VR application pipeline (e.g. to restrict latency). Our guidelines are principally aimed towards helping designers to elicit embodiment during manipulation tasks, while also providing 
Table 1: Table recapitulating the proposed guidelines and the corresponding sections to justify these guidelines

\begin{tabular}{|l|l|l|}
\hline \multicolumn{2}{|l|}{ Input Devices } \\
\hline ID1 & For specific applications or training, use an input device adapted to the task & Section 4.1.1 \\
\hline ID2 & Prioritise full-hand tracking when possible & Section 4.1 .3 \\
\hline \multicolumn{2}{|l|}{ Control } & Section 4.2.1 \\
\hline CM1 & For wide and fast motions, do not apply constraints & Section 4.2.2 \\
\hline CM2 & For wide and fast motions, choose a CD gain equal to 1 & Section 4.2.4 \\
\hline CM3 & For small motion and precise manipulation, provide assistance to the user's movements & Section 4.2 .4 \\
\hline CM4 & For precise manipulation, handle collisions & Section 4.2.2 \\
\hline CM5 & Progressively increase the distortion gain to make it more acceptable & Section 3.1 \\
\hline \multicolumn{2}{|l|}{} & Section 4.3 .2 \\
\hline FB1 & Provide a well-calibrated avatar & Sections 3.2 and 4.3.1 \\
\hline FB2 & Use first-person perspective & Section 4.2.3 \\
\hline FB3 & Use slightly transparent hands to prevent occlusions & Section 3.4 \\
\hline FB4 & Preserve body continuity as much as possible & Section 4.3 .3 \\
\hline FB5 & Maximize multisensory coherency & Section 3.3 \\
\hline FB6 & Provide haptic feedback & \\
\hline FB7 & Use realistic avatars when using distorted motion & \\
\hline
\end{tabular}

insights when a trade-off between embodiment and performance is required.

The guidelines (see in Table 1) are classified into three parts: input devices, control and feedback.

\subsection{Input Devices}

ID1: For specific applications or training, use an input device adapted to the task. For applications training the user on the use of a specific tool, use an adapted device resembling the real tool. Place the 3D model of the device in the avatar's hand (see guideline FB5). Using an adequate device for a task yields better performance [79]. This is essential for example when learning technical gestures, but otherwise generic controllers can be used.

ID2: Prioritise full-hand tracking when possible. Use hand tracking technologies (Leap Motion, gloves) instead of controllers when the tracking works well. In case of tracking issues, consider the use of 6-DoF controllers, especially controllers with capacitive sensors for the fingers. Using gestures and direct manipulation is preferred to ensure embodiment, realism, enjoyment, but only as long as the tracking is sufficient $[59,72]$. Six-DoF controllers still yield good levels of embodiment in most experiments.

\subsection{Control and mapping}

CM1: For wide and fast motions, do not apply constraints. When wide and fast movements are done by the user, do not apply constraints (such as avoiding collisions or maintaining a hand pose on an object). Constraints might create a noticeable mismatch, decreasing the sense of self-location and potentially the sense of agency $[55,86]$.

CM2: For wide and fast motions, choose a $\mathrm{CD}$ gain equal to 1. For wide motion, the best is to keep a gain equal to 1. A gain lower than 1 could be perceived as latency. A higher gain can quickly create huge offsets between the real hand and the virtual hand, affecting the sense of embodiment [55].

CM3: For small motion and precise manipulation, provide assistance to the user's movements. Use CD gains lower than 1 to precisely approach an object and/or automatic realistic poses to assist the user in their precise movements. It is possible to use subtle modifications of the motion without disturbing the sense of embodiment [32]. This must be used as assistance to provide a visually realistic hand pose to the user during manipulation.

CM4: For precise manipulation, handle collisions. Constrain the avatar's hand and fingers outside of virtual objects during manipulation. Users are more sensitive to collisions than a small mismatch between the position of their virtual and that of their real one. Therefore, they prefer seeing their virtual hand outside of objects [20].

CM5: Progressively increase the distortion gain to make it more acceptable. The $C D$ gain does not have to be constant, it can evolve during the application. Decrease it slowly to help precise manipulation. Apply a gain to avoid tiring motion, and then set it back to 1 . It was found that distorted motions are better accepted when the distortion is gradually introduced [26, 81]. In this case, the sense of embodiment is less impacted.

\subsection{Feedback}

FB1: Provide a well-calibrated avatar. The avatar's body measurements (height, general scale, arm length) must match those of the user. Eye height is important to estimate object size [58]. Having a personalised hand size was found better for manipulating objects and estimating their size $[47,114]$.

FB2: Use first-person perspective. The viewpoint should be co-located with that of the avatar. First-person perspective is better 
both for accuracy in manipulation tasks and for the sense of ownership [37]. This was already recommended by Gabbard to increase the sense of presence [31].

FB3: Use slightly transparent hands to prevent occlusions. Make the hand subtly transparent to avoid occlusions and still provide depth cues. It can become transparent when close to an object. While Gabbard already proposed this guideline for selection (see guideline Select7 [31]), we want to highlight here that a slightly transparent hand can still elicit a sense of agency [16] and ownership [64].

FB4: Preserve body continuity as much as possible. If anisomorphic motion is used for out-of-reach manipulation (Go-Go technique), use feedback such as a long arm illusion or a robotic arm, but do not break body continuity. Avoid a too high offset that would make the arm too long. Breaking body continuity during interaction can result in a loss of embodiment [96]. However, if the chosen feedback is a long arm, the embodiment can break when the arm reaches twice the length of the normal arm [51]. In this case, other types of feedback could be used, like a "ghost" hand [116] that manipulates the remote object instead of the real hand. Raycasting can also be a good alternative since it is more efficient, albeit less intuitive [84].

FB5: Maximize multisensory coherency. If a controller is used, display its $3 D$ model into the avatar's hand. It is if the real hand pose is similar to the avatar's hand pose. It also provides coherency between visual and passive haptic feedback [44]. If various virtual tools are used in the application, new input devices have been invented to propose a passive haptic feedback adapted to the virtual tool [118].

FB6: Provide haptic feedback. Use devices that provide haptic feedback during manipulation.

Haptic feedback can increase the sense of embodiment [30]. It can also help to improve performance when hand tracking is used [72].

FB7: Use realistic avatars when using distorted motion. Use realistic avatars to make distorted motion less noticeable by the user. One condition for this is to have good hand tracking. They have several advantages as they make remapped movements less noticeable [75] and increase the sense of ownership [113]. However, realistic avatars increase users' expectations in terms of control [4], therefore they should mostly be used when the tracking works well.

\section{RESEARCH TOPICS}

This section complements the previous guidelines by presenting the current gaps in the literature and proposing a number of research topics that should be explored in the future. Most of these research topics arise from the fact that the design of manipulation techniques rarely takes the avatar into account in the design process. While several works already justify design guidelines, the analysis of the different issues in Section 3 and Section 4 showed several unexplored leads. In this part, we highlight several research topics (RT) that we believe should be investigated further. These questions are focused on manipulation tasks and their interrelationships with avatars. This completes the lines of research on user representation proposed by Seinfeld et al. [95].

RT1: Study perception of motion alterations using different user representations.

Ogawa et al. [75] compared the detection thresholds for a spheric cursor and a realistic hand, and found that distorted motion was less noticeable with a realistic hand. More of this kind of studies need to be done to investigate the role of avatars on the detection of distorted motion. It is necessary to understand how to safely modify motion, without the user noticing it. In addition to detection thresholds, motion alterations can influence how the users' perceive the task. For example, several works have shown that changes in the CD gain could elicit illusions of weight when lifting virtual objects [45].

RT2: Study different possibilities of feedback and mappings of controller-based input when an avatar is involved.

The perceived sense of embodiment towards the virtual representation highly depends on the mapping and feedback used, based on the information provided by the input device. While there are several studies on hand tracking, there is a lack of investigation on the visual feedback of controller-based input [61]. Different mappings for a chosen input device should also be tested.

RT3: Compare different input devices to control the avatar during manipulation.

There is an increasing number of controllers proposed that should be compared with one another. For one user representation, several devices can be used to control it [2]. The type of input device was found to both affect interaction efficiency and embodiment [59]. It would be interesting to compare controllers allowing fingers movement (e.g. Valve Index controllers) to controllers that need to be grabbed (e.g. Vive controllers).

RT4: Propose new techniques to separate axes during manipulation while maintaining embodiment.

Constraining manipulation is sometimes needed for the sake of accuracy. For example, the user might want to move an object on a plane, or on an axis. Inspired by 3D desktop interfaces using widgets, similar techniques have been invented for immersive $\mathrm{VE}$ [70]. If the user interacts with these widgets via an avatar, additional occlusions and clutching problems can appear. If the avatar does not correctly hold the widget, the feedback can seem unrealistic and disturb embodiment. If the movement is only constrained on a certain axis, users might feel a loss of agency, so it does not seem like a good solution either. It could be interesting to imagine new techniques to manipulate objects independently on different axes without affecting embodiment.

RT5: Compare different visual feedback for common techniques.

Different visual feedback can be provided for one chosen technique. For unrealistic interaction, it may be better to provide virtual tools that suggest the use of the technique. For example, putting a laser pointer in the avatar's hand to use raycasting. In some cases, the avatar can be adapted to the technique (long arm for the Go-Go), 
but this modifies the avatar appearance and may impact more the sense of embodiment. The impact of visual feedback has been investigated for the Go-Go technique [25]. Yet, there is a need for more similar studies testing different feedback and their impact on performance and embodiment. Another solution could be to decouple the avatar from the tool used for manipulation. When using anisomorphic manipulation, the transformations could be applied only on the tool, not the avatar. This type of feedback would not affect the avatar itself, thus potentially preserving embodiment.

RT6: Study the influence of visual feedback during grasping (collisions, hand poses) on embodiment, especially on the sense of agency.

Nowadays, frameworks (e.g. SteamVR) make it easy to define hand poses for object manipulation, to have a more realistic visual feedback. Users can be guided to defined poses with subtle assistance, with no impact on embodiment [81]. They are biased to self-attribute assisted movements [32]. However, overly simplistic mappings during grasping might make the user feel less in control. New solutions start to be proposed to automatically compute hand poses in real time [109] that could potentially improve embodiment.

RT7: Explore the impact of multimodal sensory feedback during manipulation on the sense of embodiment.

While there are studies showing the importance of synchronous multimodal feedback on the sense of embodiment, there is a lack of literature on the importance of haptic and auditory feedback, especially during interaction tasks. Yet, the potential impact of such feedback was found on body perception [30, 106]. Manipulating objects offers a source of potential feedback that might increase the sense of embodiment.

We hope that these proposed research topics can inspire researchers in VR and HCI community, and lead to new ways of designing interaction techniques with avatars in the future.

\section{RETHINK EVALUATION METHODS}

In this paper we propose guidelines for early design phases. As avatars can impact interaction (as detailed in Section 3, e.g. influencing users' speed [111]), we believe that it is becoming important to also consider evaluating the influence of the avatar throughout the whole design process, as well as to consider novel evaluation methods assessing the compatibility between an avatar and an in teraction technique. This of course does not need to be limited to manipulation techniques, but can be extended to all types of interaction techniques. For instance, avatars start to be considered in the evaluation of navigation [68] or system input techniques [38, 52]. In this part, we present several directions that we believe should be explored in terms of evaluation to reach this goal.

\subsection{Do Not Disregard the Role of the Avatar During the Evaluation Process}

Several studies have shown that avatar characteristics can have an impact on user performance [90,111]. These studies mostly investigated the effect of avatar appearance (e.g. visual hand representation versus tool representation only [90], hand versus whole arm [111]) or of the DoF offered by the representation (e.g. sphere versus hand with finger control [4]). However, the limited number of studies exploring these effects also highlighted a need for further investigating this impact depending on the task performed. Each avatar offers a certain level of control to the user, therefore the choice of a representation might also depend on the complexity of the task to perform. A hand representation may be more suitable for precise manipulation, while a sphere may be sufficient for rough object selection. This potential impact of avatars must therefore be considered in the design process, and designers should try different user representations early on in the development process to check that they do not impact interaction.

\subsection{Use Embodiment as a Novel Criterion for Assessing the Quality of Interaction}

During iterations of the design process, different types of evaluation are typically performed, where designers use several criteria and heuristics to identify flaws in a current implementation (formative evaluation) or to compare a new final implementation to benchmarks or older implementations (summative evaluation). These criteria are mostly performance-oriented (e.g. accuracy, speed) or user-oriented (e.g. ease of use, predictability). Given the literature now available in regards to the sense of embodiment, we believe that it should progressively become a new criterion for measuring the quality of interaction, just as breaks in presence were already proposed as new criteria to measure usability [101]. In the future, the goal would therefore be to design efficient interaction techniques that do not disrupt embodiment, in which case questions related to the sense of embodiment $[36,91]$ should be added to formative and summative evaluations to complete evaluations during the iterative design process.

\subsection{Improve Embodiment Measurements}

While the sense of virtual embodiment is typically assessed through subjective questionnaires, finding novel and more appropriate means of evaluating the sense of embodiment is an on-going research topic. For instance, the need for more standardised evaluations of embodiment has been identified [36]. To this end, Roth and Latoschik [91] recently constructed a novel embodiment questionnaire by relying on factor analysis to propose new leads of standardised questions. Interestingly, their work brought out a novel dimension to evaluate embodiment, named Change (in the perceived body schema), which was not present in the subcomponents proposed by Kilteni et al. [50], suggesting that novel dimensions might emerge from the formal construction of novel questionnaires, and which could be more related to interaction. Nevertheless, embodiment evaluations currently mostly rely on this type of subjective questionnaires, as the proprioceptive drift originally used as an embodiment measure in the rubber-hand illusion [12] cannot be used anymore with avatars seen from a first-person perspective. Other approaches to evaluate the sense of embodiment rely on behavioural responses, such as threat responses, which were found to be correlated with embodiment [117]. However, the introduction of threat can potentially change users' behaviour [29] and it is not 
a precise and direct measure as serious threat response and embodiment can be sometimes dissociated [63]. In the future we may be able to use more precise methods to measure embodiment. For instance, EEG measures are an interesting path that is beginning to be explored in the context of embodiment [46]. In particular, it was recently investigated while controlling a walking avatar [1], which opens new questions in terms of interaction tasks in general.

\section{CONCLUSION}

In this paper, we discussed design challenges emerging when the user is represented by an avatar during manipulation tasks, with the goal of identifying practical advice for developers and stimulating reflection and research on this topic. We proposed a number of guidelines to design avatar-friendly techniques, as well as a number of research topics demonstrating the need for further investigation. We hope that in the future, thanks to deeper research on this topic, we will be able to reach good trade-offs ensuring embodiment and efficient manipulation. Finally, the avatar-friendly concept can be extended to other types of interaction (navigation, system control) to provide vibrant 3D experiences to users.

\section{ACKNOWLEDGMENTS}

We wish to thank all the reviewers for their constructive comments. This work was sponsored by the Inria Research Challenge Avatar. We would also like to thank Thomas Howard for his help with proofreading and corrections.

\section{REFERENCES}

[1] Bilal Alchalabi, Jocelyn Faubert, and David R. Labbe. 2019. EEG Can Be Used to Measure Embodiment When Controlling a Walking Self-Avatar. In 2019 IEEE Conference on Virtual Reality and 3D User Interfaces (VR). 776-783. https: //doi.org/10.1109/VR.2019.8798263

[2] Ayman Alzayat, Mark Hancock, and Miguel A. Nacenta. 2019. Quantitative Measurement of Tool Embodiment for Virtual Reality Input Alternatives. In Proceedings of the 2019 CHI Conference on Human Factors in Computing Systems (Glasgow, Scotland Uk) (CHI '19). 1-11.

[3] Ferran Argelaguet and Carlos Andujar. 2013. A survey of 3D object selection techniques for virtual environments. Computers \& Graphics 37, 3 (2013), 121136.

[4] Ferran Argelaguet, Ludovic Hoyet, Michael Trico, and Anatole Lécuyer. 2016. The role of interaction in virtual embodiment: Effects of the virtual hand representation. In 2016 IEEE Virtual Reality (VR). 3-10.

[5] Elena Azañón, Luigi Tamè, Angelo Maravita, Sally A Linkenauger, Elisa R Ferrè, Ana Tajadura-Jiménez, and Matthew R Longo. 2016. Multimodal contributions to body representation. Multisensory research 29, 6-7 (2016), 635-661.

[6] Mahdi Azmandian, Mark Hancock, Hrvoje Benko, Eyal Ofek, and Andrew D. Wilson. 2016. Haptic Retargeting: Dynamic Repurposing of Passive Haptics for Enhanced Virtual Reality Experiences. In Proceedings of the 2016 CHI Conference on Human Factors in Computing Systems (CHI '16). 1968-1979.

[7] Domna Banakou, Sameer Kishore, and Mel Slater. 2018. Virtually Being Einstein Results in an Improvement in Cognitive Task Performance and a Decrease in Age Bias. Frontiers in Psychology 9 (2018), 917.

[8] Anil Batmaz, Aunnoy Mutasim, and Wolfgang Stuerzlinger. 2020. Precision vs. Power Grip: A Comparison of Pen Grip Styles for Selection in Virtual Reality. In Workshop on Novel Input Devices and Interaction Techniques (NIDIT) at IEEE VR 2020.

[9] Ayush Bhargava, Jeffrey W. Bertrand, Anand K. Gramopadhye, Kapil C. Madathil, and Sabarish V. Babu. 2018. Evaluating Multiple Levels of an Interaction Fidelity Continuum on Performance and Learning in Near-Field Training Simulations. IEEE Transactions on Visualization and Computer Graphics 24, 4 (2018), 14181427

[10] Christoph Bichlmeier, Sandro Michael Heining, Marco Feuerstein, and Nassir Navab. 2009. The Virtual Mirror: A New Interaction Paradigm for Augmented Reality Environments. IEEE Transactions on Medical Imaging 28, 9 (2009), 14981510 .

[11] Richard A. Bolt. 1980. "Put-That-There": Voice and Gesture at the Graphics Interface. SIGGRAPH Comput. Graph. 14, 3 (1980), 262-270.
[12] Matthew Botvinick and Jonathan Cohen. 1998. Rubber hands 'feel'touch that eyes see. Nature 391, 6669 (1998), 756-756.

[13] Pierre Bourdin, Matteo Martini, and Maria V Sanchez-Vives. 2019. Altered visual feedback from an embodied avatar unconsciously influences movement amplitude and muscle activity. Scientific Reports 9, 1 (2019), 1-9.

[14] Doug A Bowman. 1999. Interaction techniques for common tasks in immersive virtual environments: design, evaluation, and application. Ph.D. Dissertation. Georgia Institute of Technology.

[15] Doug A. Bowman, Ernst Kruijff, Joseph J. LaViola, and Ivan Poupyrev. 2004. 3D User Interfaces: Theory and Practice. Addison Wesley Longman Publishing Co., Inc., USA.

[16] Volkert Buchmann, Trond Nilsen, and Mark Billinghurst. 2005. Interaction with Partially Transparent Hands and Objects. In Proceedings of the Sixth Australasian Conference on User Interface - Volume 40 (AUIC '05). Australian Computer Society, Inc., AUS, 17-20.

[17] Grigore C Burdea and Frederick P Brooks. 1996. Force and touch feedback for virtual reality. (1996).

[18] Dalila Burin, Konstantina Kilteni, Marco Rabuffetti, Mel Slater, and Lorenzo Pia. 2019. Body ownership increases the interference between observed and executed movements. PloS one 14, 1 (2019).

[19] Eric Burns, Sharif Razzaque, Abigail T. Panter, Mary C. Whitton, Matthew R. McCallus, and Frederick P. Brooks. 2005. The hand is slower than the eye: a quantitative exploration of visual dominance over proprioception. In IEEE Proceedings. VR 2005. Virtual Reality, 2005. 3-10.

[20] Ryan Canales, Aline Normoyle, Yu Sun, Yuting Ye, Massimiliano Di Luca, and Sophie Jörg. 2019. Virtual Grasping Feedback and Virtual Hand Ownership. In ACM Symposium on Applied Perception 2019 (SAP '19). Article 4, 9 pages.

[21] Géry Casiez, Daniel Vogel, Ravin Balakrishnan, and Andy Cockburn. 2008. The impact of control-display gain on user performance in pointing tasks. Humancomputer interaction 23, 3 (2008), 215-250.

[22] Lionel Dominjon, Anatole Lecuyer, Jean-Marie Burkhardt, Paul Richard, and Simon Richir. 2005. Influence of control/display ratio on the perception of mass of manipulated objects in virtual environments. In IEEE Proceedings. VR 2005. Virtual Reality, 2005. 19-25.

[23] Mark Draper. 1995. Exploring the influence of a virtual body on spatial awareness. Master's thesis. University of Washington.

[24] Shaghayegh Esmaeili, Brett Benda, and Eric D Ragan. 2020. Detection of Scaled Hand Interactions in Virtual Reality: The Effects of Motion Direction and Task Complexity. In 2020 IEEE Conference on Virtual Reality and 3D User Interfaces (VR). IEEE, 453-462.

[25] Tiare Feuchtner and Jörg Müller. 2017. Extending the Body for Interaction with Reality. In Proceedings of the 2017 CHI Conference on Human Factors in Computing Systems (CHI '17). 5145-5157.

[26] Tiare Feuchtner and Jörg Müller. 2018. Ownershift: Facilitating Overhead Interaction in Virtual Reality with an Ownership-Preserving Hand Space Shift. In Proceedings of the 31st Annual ACM Symposium on User Interface Software and Technology (UIST '18). 31-43.

[27] Scott Frees and G.D. Kessler. 2005. Precise and rapid interaction through scaled manipulation in immersive virtual environments. Proceedings - IEEE Virtual Reality, 99-106.

[28] Rebecca Fribourg, Ferran Argelaguet, Anatole Lécuyer, and Ludovic Hoyet. 2020. Avatar and Sense of Embodiment: Studying the Relative Preference Between Appearance, Control and Point of View. IEEE Transactions on Visualization and Computer Graphics 26, 5 (2020), 2062-2072.

[29] Rebecca Fribourg, Evan Blanpied, Ludovic Hoyet, Anatole Lécuyer, and Ferran Argelaguet Sanz. 2020. Influence of Threat Occurrence and Repeatability on the Sense of Embodiment and Threat Response in VR. In International Conference on Artificial Reality and Telexistence \& Eurographics Symposium on Virtual Environments (ISMAR). 9p

[30] Jakob Fröhner, Gionata Salvietti, Philipp Beckerle, and Domenico Prattichizzo. 2019. Can Wearable Haptic Devices Foster the Embodiment of Virtual Limbs? IEEE Transactions on Haptics 12, 3 (2019), 339-349.

[31] Joseph L. Gabbard. 1998. A Taxonomy of Usability Characteristics in Virtual Environments. master's thesis,. Dept. of Computer Science and Applications, Virginia Polytechnic Institute and State University.

[32] Henrique Galvan Debarba, Ronan Boulic, Roy Salomon, Olaf Blanke, and Bruno Herbelin. 2018. Self-attribution of distorted reaching movements in immersive virtual reality. Computers \& Graphics 76 (2018), 142 - 152.

[33] Henrique Galvan Debarba, Sidney Bovet, Roy Salomon, Olaf Blanke, Bruno Herbelin, and Ronan Boulic. 2017. Characterizing first and third person viewpoints and their alternation for embodied interaction in virtual reality. PLOS ONE 12, 12 (2017), 1-19.

[34] BoYu Gao, Joonwoo Lee, Huawei Tu, Wonjun Seong, and HyungSeok Kim. 2020. The Effects of Avatar Visibility on Behavioral Response with or without MirrorVisual Feedback in Virtual Environments. In 2020 IEEE Conference on Virtual Reality and 3D User Interfaces Abstracts and Workshops (VRW). 780-781. 
[35] Paul Christopher Gloumeau, Wolfgang. Stuerzlinger, and JungHyun Han. 2020 PinNPivot: Object Manipulation using Pins in Immersive Virtual Environments. IEEE Transactions on Visualization and Computer Graphics (2020), 1-1.

[36] Mar González-Franco and Tabitha C. Peck. 2018. Avatar Embodiment. Towards a Standardized Questionnaire. Frontiers in Robotics and AI 5 (2018), 74.

[37] Geoffrey Gorisse, Olivier Christmann, Etienne Armand Amato, and Simon Richir. 2017. First- and Third-Person Perspectives in Immersive Virtual Environments: Presence and Performance Analysis of Embodied Users. Frontiers in Robotics and $A I 4$ (2017), 33

[38] Jens Grubert, Lukas Witzani, Eyal Ofek, Michel Pahud, Matthias Kranz, and Per Ola Kristensson. 2018. Effects of Hand Representations for Typing in Virtual Reality. In 2018 IEEE Conference on Virtual Reality and 3D User Interfaces (VR) $151-158$.

[39] Edward T. Hall. 1966. The hidden dimension. Vol. 609. Garden City, NY: Doubleday.

[40] Devamardeep Hayatpur, Seongkook Heo, Haijun Xia, Wolfgang Stuerzlinger and Daniel Wigdor. 2019. Plane, Ray, and Point: Enabling Precise Spatial Manipulations with Shape Constraints. In Proceedings of the 32nd Annual ACM Symposium on User Interface Software and Technology (UIST '19). 1185-1195.

[41] Koichi Hirota and Michitaka Hirose. 1995. Providing force feedback in virtual environments. IEEE Computer Graphics and Applications 15, 5 (1995), 22-30.

[42] Ludovic Hoyet, Ferran Argelaguet, Corentin Nicole, and Anatole Lécuyer. 2016. "Wow! I Have Six Fingers!" Would You Accept Structural Changes of Your Hand in VR? Frontiers in Robotics and AI 3 (2016), 27.

[43] Yi-Jheng Huang, Kang-Yi Liu, Suiang-Shyan Lee, and I-Cheng Yeh. 2020. Evaluation of a Hybrid of Hand Gesture and Controller Inputs in Virtual Reality. International fournal of Human-Computer Interaction 0, 0 (2020), 1-12.

[44] Brent Edward Insko, M Meehan, M Whitton, and F Brooks. 2001. Passive haptics significantly enhances virtual environments. Ph.D. Dissertation. University of North Carolina at Chapel Hill.

[45] David A. G. Jauregui, Ferran Argelaguet, Anne-Hélène Olivier, Maud Marchal, Franck Multon, and Anatole Lecuyer. 2014. Toward "Pseudo-Haptic Avatars": Modifying the Visual Animation of Self-Avatar Can Simulate the Perception of Weight Lifting. IEEE Transactions on Visualization and Computer Graphics 20, 4 (2014), 654-661.

[46] C. Jeunet, L. Albert, F. Argelaguet, and A. Lécuyer. 2018. "Do You Feel in Control?": Towards Novel Approaches to Characterise, Manipulate and Measure the Sense of Agency in Virtual Environments. IEEE Transactions on Visualization and Computer Graphics 24, 4 (2018), 1486-1495. https://doi.org/10.1109/TVCG. 2018.2794598

[47] Sungchul Jung, Gerd Bruder, Pamela J. Wisniewski, Christian Sandor, and Charles E. Hughes. 2018. Over My Hand: Using a Personalized Hand in VR to Improve Object Size Estimation, Body Ownership, and Presence. In Proceeding of the Symposium on Spatial User Interaction (Berlin, Germany) (SUI '18). 60-68.

[48] Abdelmajid Kadri, Anatole Lecuyer, Jean-Marie Burkhardt, and Simon Richir. 2007. The Visual Appearance of User's Avatar Can Influence the Manipulation of Both Real Devices and Virtual Objects. In 2007 IEEE Symposium on 3D User Interfaces.

[49] Konstantina Kilteni, Ilias Bergstrom, and Mel Slater. 2013. Drumming in immersive virtual reality: the body shapes the way we play. IEEE transactions on visualization and computer graphics 19, 4 (2013), 597-605.

[50] Konstantina Kilteni, Raphaela Groten, and Mel Slater. 2012. The Sense of Embodiment in Virtual Reality. Presence: Teleoperators and Virtual Environments 21, 4 (2012), 373-387.

[51] Konstantina Kilteni, Jean-Marie Normand, Maria V. Sanchez-Vives, and Mel Slater. 2012. Extending Body Space in Immersive Virtual Reality: A Very Long Arm Illusion. PLOS ONE 7, 7 (2012), 1-15.

[52] Pascal Knierim, Valentin Schwind, Anna Maria Feit, Florian Nieuwenhuizen, and Niels Henze. 2018. Physical Keyboards in Virtual Reality: Analysis of Typing Performance and Effects of Avatar Hands. In Proceedings of the $2018 \mathrm{CHI}$ Conference on Human Factors in Computing Systems (CHI '18). 1-9.

[53] Luv Kohli, Mary C Whitton, and Frederick P Brooks. 2012. Redirected touching: The effect of warping space on task performance. In 2012 IEEE Symposium on $3 D$ User Interfaces (3DUI). IEEE, 105-112.

[54] Elena Kokkinara and Mel Slater. 2014. Measuring the Effects through Time of the Influence of Visuomotor and Visuotactile Synchronous Stimulation on a Virtual Body Ownership Illusion. Perception 43, 1 (2014), 43-58. PMID: 24689131.

[55] Elena Kokkinara, Mel Slater, and Joan López-Moliner. 2015. The Effects of Visuomotor Calibration to the Perceived Space and Body, through Embodiment in Immersive Virtual Reality. ACM Trans. Appl. Percept. 13, 1, Article 3 (2015), 22 pages.

[56] Ki-Uk Kyung and Dong-Soo Kwon. 2006. Multi-sensory Perception of Roughness: Empirical Study on Effects of Vibrotactile Feedback and Auditory Feedback in Texture Perception. In Advances in Artificial Reality and Tele-Existence, Zhigeng Pan, Adrian Cheok, Michael Haller, Rynson W. H. Lau, Hideo Saito, and Ronghua Liang (Eds.). Springer Berlin Heidelberg, Berlin, Heidelberg, 406-415.

[57] Bigna Lenggenhager, Michael Mouthon, and Olaf Blanke. 2009. Spatial aspects of bodily self-consciousness. Consciousness and cognition 18, 1 (2009), 110-117.
[58] Markus Leyrer, Sally A. Linkenauger, Heinrich H. Bülthoff, Uwe Kloos, and Betty Mohler. 2011. The Influence of Eye Height and Avatars on Egocentric Distance Estimates in Immersive Virtual Environments. In Proceedings of the ACM SIGGRAPH Symposium on Applied Perception in Graphics and Visualization (Toulouse, France) (APGV '11). 67-74.

[59] Lorraine Lin, Aline Normoyle, Alexandra Adkins, Yu Sun, Andrew Robb, Yuting Ye, Massimiliano Di Luca, and Sophie Jörg. 2019. The Effect of Hand Size and Interaction Modality on the Virtual Hand Illusion. In 2019 IEEE Conference on Virtual Reality and 3D User Interfaces (VR). 510-518.

[60] Benjamin Lok, Samir Naik, Mary Whitton, and Frederick P. Brooks. 2003. Effects of Handling Real Objects and Self-Avatar Fidelity on Cognitive Task Performance and Sense of Presence in Virtual Environments. Presence 12, 6 (2003), 615-628.

[61] Christos Lougiakis, Akrivi Katifori, Maria Roussou, and Ioannis-Panagiotis Ioannidis. 2020. Effects of virtual hand representation on interaction and embodiment in HMD-based virtual environments using controllers. 2020 IEEE Conference on Virtual Reality and 3D User Interfaces (VR) (2020), 510-518.

[62] Jean-Luc Lugrin, David Obremski, Daniel Roth, and Marc Erich Latoschik. 2016. Audio Feedback and Illusion of Virtual Body Ownership in Mixed Reality. In Proceedings of the 22nd ACM Conference on Virtual Reality Software and Technology (Munich, Germany) (VRST '16). 309-310.

[63] Ke Ma and Bernhard Hommel. 2013. The virtual-hand illusion: effects of impact and threat on perceived ownership and affective resonance. Frontiers in psychology 4 (2013), 604.

[64] Matteo Martini, Konstantina Kilteni, Antonella Maselli, and Maria V SanchezVives. 2015. The body fades away: investigating the effects of transparency of an embodied virtual body on pain threshold and body ownership. Scientific reports 5 (2015), 13948.

[65] Ryan P. McMahan, Chengyuan Lai, and Swaroop K. Pal. 2016. Interaction Fidelity: The Uncanny Valley of Virtual Reality Interactions. In Virtual, Augmented and Mixed Reality, Stephanie Lackey and Randall Shumaker (Eds.). Springer International Publishing, Cham, 59-70.

[66] Erin A. McManus, Bobby Bodenheimer, Stephan Streuber, Stephan de la Rosa, Heinrich H. Bülthoff, and Betty J. Mohler. 2011. The Influence of Avatar (Self and Character) Animations on Distance Estimation, Object Interaction and Locomotion in Immersive Virtual Environments. In Proceedings of the ACM SIGGRAPH Symposium on Applied Perception in Graphics and Visualization (Toulouse, France) (APGV '11). 37-44.

[67] Daniel Medeiros, Rafael K dos Anjos, Daniel Mendes, João Madeiras Pereira, Alberto Raposo, and Joaquim Jorge. 2018. Keep my head on my shoulders! why third-person is bad for navigation in VR. In Proceedings of the 24th ACM Symposium on Virtual Reality Software and Technology. 1-10.

[68] Daniel Medeiros, Mauricio Sousa, Alberto Raposo, and Joaquim Jorge. 2019. Magic Carpet: Interaction Fidelity for Flying in VR. IEEE Transactions on Visualization and Computer Graphics (2019).

[69] Daniel Mendes, Fabio M. Caputo, Andrea Giachetti, Alfredo Ferreira, and Joaquim A. P. Jorge. 2019. A Survey on 3D Virtual Object Manipulation: From the Desktop to Immersive Virtual Environments. Computer Graphics Forum 38, 1 (2019), 21-45.

[70] Daniel Mendes, Filipe Relvas, Alfredo Ferreira, and Joaquim Jorge. 2016. The Benefits of DOF Separation in Mid-Air 3D Object Manipulation. In Proceedings of the 22nd ACM Conference on Virtual Reality Software and Technology (VRST '16). 261-268.

[71] Mark R. Mine. 1995. Virtual Environment Interaction Techniques. Technical Report. USA.

[72] Mathias Moehring and Bernd Froehlich. 2011. Effective manipulation of virtual objects within arm's reach. In 2011 IEEE Virtual Reality Conference. 131-138.

[73] Betty J. Mohler, Sarah H. Creem-Regehr, William B. Thompson, and Heinrich H. Bülthoff. 2010. The Effect of Viewing a Self-Avatar on Distance Judgments in an HMD-Based Virtual Environment. Presence 19, 3 (2010), 230-242.

[74] Nami Ogawa, Takuji Narumi, and Michitaka Hirose. 2018. Object Size Perception in Immersive Virtual Reality: Avatar Realism Affects the Way We Perceive. In 2018 IEEE Conference on Virtual Reality and 3D User Interfaces (VR). 647-648.

[75] Nami Ogawa, Takuji Narumi, and Michitaka Hirose. 2020. Effect of Avatar Appearance on Detection Thresholds for Remapped Hand Movements. IEEE Transactions on Visualization and Computer Graphics (2020), 1-1.

[76] Nami Ogawa, Takuji Narumi, Hideaki Kuzuoka, and Michitaka Hirose. 2020. Do You Feel Like Passing Through Walls?: Effect of Self-Avatar Appearance on Facilitating Realistic Behavior in Virtual Environments. In Proceedings of the 2020 CHI Conference on Human Factors in Computing Systems (Honolulu, HI, USA) (CHI '20). 1-14.

[77] Hiroshi Ono, Brian J Rogers, Masao Ohmi, and Mika E Ono. 1988. Dynamic Occlusion and Motion Parallax in Depth Perception. Perception 17, 2 (1988), 255-266. PMID: 3226867.

[78] Nassima Ouramdane, Samir Otmane, Frédéric Davesne, and Malik Mallem. 2006. FOLLOW-ME: a new 3D interaction technique based on virtual guides and granularity of interaction. In Proceedings of the 2006 ACM international conference on Virtual reality continuum and its applications. 137-144. 
[79] Duc-Minh Pham and Wolfgang Stuerzlinger. 2019. Is the Pen Mightier than the Controller? A Comparison of Input Devices for Selection in Virtual and Augmented Reality. In 25th ACM Symposium on Virtual Reality Software and Technology (VRST '19). Article 35, 11 pages.

[80] Jodie M. Plumert, Joseph K. Kearney, James F. Cremer, and Kara Recker. 2005 Distance Perception in Real and Virtual Environments. ACM Trans. Appl. Percept. 2, 3 (2005), 216-233.

[81] Thibault Porssut, Bruno Herbelin, and Ronan Boulic. 2019. Reconciling Being in-Control vs. Being Helped for the Execution of Complex Movements in VR In 2019 IEEE Conference on Virtual Reality and 3D User Interfaces (VR). 529-537.

[82] Ivan Poupyrev. 2000. 3d manipulation techniques. 3D User Interface Design, Lecture Slides. SIGGRAPH 2000 (2000).

[83] Ivan Poupyrev, Mark Billinghurst, Suzanne Weghorst, and Tadao Ichikawa. 1996 The Go-Go Interaction Technique: Non-Linear Mapping for Direct Manipulation in VR. In Proceedings of the 9th Annual ACM Symposium on User Interface Software and Technology (UIST' '96). 79-80.

[84] Ivan Poupyrev, Tadao Ichikawa, Suzanne Weghorst, and Mark Billinghurst. 1998. Egocentric Object Manipulation in Virtual Environments: Empirical Evaluation of Interaction Techniques. Computer Graphics Forum 17, 3 (1998), 41-52.

[85] Mores Prachyabrued and Christoph W. Borst. 2012. Visual interpenetration tradeoffs in whole-hand virtual grasping. In 2012 IEEE Symposium on 3D User Interfaces (3DUI). 39-42.

[86] Stephen C. Pritchard, Regine Zopf, Vince Polito, David M. Kaplan, and Mark A Williams. 2016. Non-hierarchical Influence of Visual Form, Touch, and Position Cues on Embodiment, Agency, and Presence in Virtual Reality. Frontiers in Psychology 7 (2016), 1649.

[87] Dominika Radziun and H Henrik Ehrsson. 2018. Auditory cues influence the rubber-hand illusion. Journal of Experimental Psychology: Human Perception and Performance 44, 7 (2018), 1012

[88] Austin Rallis, Kelene A. Fercho, Taylor J. Bosch, and Lee A. Baugh. 2018. Getting a handle on virtual tools: An examination of the neuronal activity associated with virtual tool use. Neuropsychologia 109 (2018), $208-221$

[89] Natasha Ratcliffe and Roger Newport. 2017. The Effect of Visual, Spatial and Temporal Manipulations on Embodiment and Action. Frontiers in Human Neuroscience 11 (2017), 227

[90] Aylen Ricca, Amine Chellali, and Samir Otmane. 2020. Influence of hand visualization on tool-based motor skills training in an immersive VR simulator. In 19th IEEE International Symposium on Mixed and Augmented Reality (ISMAR 2020).

[91] Daniel Roth and Marc Erich Latoschik. 2020. Construction of the Virtual Embodiment Questionnaire (VEQ). IEEE Transactions on Visualization \& Computer Graphics 26, 12 (dec 2020), 3546-3556.

[92] Patrick Salamin, Daniel Thalmann, and Frédéric Vexo. 2006. The Benefits of Third-Person Perspective in Virtual and Augmented Reality? In Proceedings of the ACM Symposium on Virtual Reality Software and Technology (VRST '06). 27-30.

[93] Patrick Salamin, Daniel Thalmann, and Frédéric Vexo. 2008. Improved ThirdPerson Perspective: A Solution Reducing Occlusion of the 3PP?. In Proceedings of The 7th ACM SIGGRAPH International Conference on Virtual-Reality Continuum and Its Applications in Industry (VRCAI '08). Article 30, 6 pages.

[94] Valentin Schwind, Sven Mayer, Alexandre Comeau-Vermeersch, Robin Schweigert, and Niels Henze. 2018. Up to the Finger Tip: The Effect of Avatars on Mid-Air Pointing Accuracy in Virtual Reality. In Proceedings of the 2018 Annual Symposium on Computer-Human Interaction in Play (Melbourne, VIC, Australia) (CHI PLAY '18). 477-488.

[95] Sofia Seinfeld, Tiare Feuchtner, Antonella Maselli, and Jörg Müller. 2020. User Representations in Human-Computer Interaction. Human-Computer Interaction 0,0 (2020), 1-39.

[96] Sofia Seinfeld and Jörg Müller. 2020. Impact of Visuomotor Feedback on the Embodiment of Virtual Hands Detached from the Body. arXiv:2002.12020 [cs.HC]

[97] Irene Senna, Angelo Maravita, Nadia Bolognini, and Cesare V. Parise. 2014. The Marble-Hand Illusion. PLOS ONE 9, 3 (2014), 1-6.

[98] Mel Slater, Daniel Pérez Marcos, Henrik Ehrsson, and Maria V Sanchez-Vives. 2008. Towards a digital body: the virtual arm illusion. Frontiers in human neuroscience 2 (2008), 6.

[99] Bernhard Spanlang, Jean-Marie Normand, David Borland, Konstantina Kilteni, Elias Giannopoulos, Ausiàs Pomés, Mar González-Franco, Daniel Perez-Marcos,
Jorge Arroyo-Palacios, Xavi Navarro Muncunill, and Mel Slater. 2014. How to Build an Embodiment Lab: Achieving Body Representation Illusions in Virtual Reality. Frontiers in Robotics and AI 1 (2014), 9.

[100] Bernhard Spanlang, Jean-Marie Normand, Elias Giannopoulos, and Mel Slater. 2010. A First Person Avatar System with Haptic Feedback. In Proceedings of the 17th ACM Symposium on Virtual Reality Software and Technology (Hong Kong) (VRST '10). 47-50.

[101] Anthony Steed, Andrea Brogni, and Vinoba Vinayagamoorthy. 2005. Breaks in presence as usability criteria. In Proceedings of HCI international.

[102] Hemant Bhaskar Surale, Aakar Gupta, Mark Hancock, and Daniel Vogel. 2019. TabletInVR: Exploring the Design Space for Using a Multi-Touch Tablet in Virtual Reality. In Proceedings of the 2019 CHI Conference on Human Factors in Computing Systems (Glasgow, Scotland Uk) (CHI '19). 1-13.

[103] Alistair Sutcliffe and Brian Gault. 2004. Heuristic evaluation of virtual reality applications. Interacting with computers 16, 4 (2004), 831-849.

[104] David Swapp, Vijay Pawar, and Céline Loscos. 2006. Interaction with co-located haptic feedback in virtual reality. Virtual Reality 10, 1 (2006), 24-30.

[105] Ana Tajadura-Jiménez, Domna Banakou, Nadia Bianchi-Berthouze, and Mel Slater. 2017. Embodiment in a child-like talking virtual body influences object size perception, self-identification, and subsequent real speaking. Scientific Reports 7, 1 (2017), 1-12.

[106] Ana Tajadura-Jiménez, Manos Tsakiris, Torsten Marquardt, and Nadia BianchiBerthouze. 2015. Action sounds update the mental representation of arm dimension: contributions of kinaesthesia and agency. Frontiers in Psychology 6 (2015), 689

[107] Vildan Tanriverdi and Robert J.K. Jacob. 2001. VRID: A Design Model and Methodology for Developing Virtual Reality Interfaces. In Proceedings of the ACM Symposium on Virtual Reality Software and Technology (Baniff, Alberta, Canada) (VRST '01). 175-182.

[108] Robert J. Teather and Wolfgang Stuerzlinger. 2011. Pointing at 3D targets in stereo head-tracked virtual environment. In 2011 IEEE Symposium on 3D User Interfaces (3DUI). 87-94.

[109] Hao Tian, Changbo Wang, Dinesh Manocha, and Xinyu Zhang. 2019. Realtime Hand-Object Interaction Using Learned Grasp Space for Virtual Environments. IEEE Transactions on Visualization and Computer Graphics 25, 8 (2019), 26232635 .

[110] Gaetano Tieri, Emmanuele Tidoni, Enea Francesco Pavone, and Salvatore Maria Aglioti. 2015. Body visual discontinuity affects feeling of ownership and skin conductance responses. Scientific reports 5 (2015), 17139.

[111] Tanh Quang Tran, HyunJu Shin, Wolfgang Stuerzlinger, and JungHyun Han. 2017. Effects of Virtual Arm Representations on Interaction in Virtual Environments. In Proceedings of the 23rd ACM Symposium on Virtual Reality Software and Technology (VRST '17). Article 40, 9 pages.

[112] Manos Tsakiris, Gita Prabhu, and Patrick Haggard. 2006. Having a body versus moving your body: How agency structures body-ownership. Consciousness and cognition 15, 2 (2006), 423-432.

[113] Thomas Waltemate, Dominik Gall, Daniel Roth, Mario Botsch, and Marc E. Latoschik. 2018. The Impact of Avatar Personalization and Immersion on Virtual Body Ownership, Presence, and Emotional Response. IEEE Transactions on Visualization and Computer Graphics 24, 4 (2018), 1643-1652.

[114] Yanqing Wang, Christine L. MacKenzie, and Valerie A. Summers. 1997. Object Manipulation in Virtual Environments: Human Bias, Consistency and Individual Differences. In CHI '97 Extended Abstracts on Human Factors in Computing Systems (Atlanta, Georgia) (CHI EA '97). 349-350.

[115] Chadwick A. Wingrave and Joseph J. LaViola. 2010. Reflecting on the Design and Implementation Issues of Virtual Environments. Presence 19, 2 (2010), 179-195.

[116] Ungyeon Yang and Gerard Jounghyun Kim. 2002. Implementation and Evaluation of "Just Follow Me": An Immersive, VR-Based, Motion-Training System. Presence: Teleoperators and Virtual Environments 11, 3 (2002), 304-323.

[117] Ye Yuan and Anthony Steed. 2010. Is the rubber hand illusion induced by immersive virtual reality?. In 2010 IEEE Virtual Reality Conference (VR). IEEE, 95-102.

[118] André Zenner and Antonio Krüger. 2017. Shifty: A Weight-Shifting Dynamic Passive Haptic Proxy to Enhance Object Perception in Virtual Reality. IEEE Transactions on Visualization and Computer Graphics 23, 4 (2017), 1285-1294.

[119] Shumin Zhai, William Buxton, and Paul Milgram. 1994. The "Silk Cursor": Investigating Transparency for 3D Target Acquisition. In Proceedings of the SIGCHI Conference on Human Factors in Computing Systems (CHI '94). 459-464. 